Ann. Biol. anim. Bioch. Biophys., 1978, 18 (4), 805-811.

\title{
Effet d'hormones gonadotropes, in vitro, sur la concentration de l'adénosine monophosphate cyclique dans l'ovaire de l'anguille (Anguilla anguilla L.)
}

\author{
par Elizabeth FONTAINE-BERTRAND, C. SALMON, Y. A. FONTAINE \\ arec la collaboration technique de Nadine DELERUE-LE-BELLE et J. MARCHELIDON. \\ Laboratoire de Physiologie générale el comparée du Muséum \\ national d'Histoire naturelle, Laboratoire d'Endocrinologie \\ comparée associé au CNRS, 7 rue Cuvier, 75005 Paris.
}

Summary. Cyclic adenosine monophosphate content in the ovary of the eel (Anguilla anguilla L.). Effect of gonadotropic hormones in vitro.

Freshwater european eels never reach sexual maturity naturally but injecting carp gonadotropin (c-GTH) induced sexual development. In vitro, the incubation of pieces of silver eel ovary with c-GTH increased c-AMP levels. The size of the increase depended both on incubation time and hormone concentration (from 0.01 to $10 \mu \mathrm{g} / \mathrm{ml}$ ) and the response could be used as a gonadotropin bioassay. Relative to c-GTH, the GTH from another Teleost, the indian catfish had the same potency in increasing c-AMP concentration in the eel ovary as in inducing spermiation in the frog. On the other hand, the two assays gave very different results in the case of the GTH isolated from a Chondrostean, the sturgeon. The ovaries from c-GTH-treated silver eels, which had undergone some development, showed a smaller in vifro response to gonadotropin than did ovaries from untreated eels. Yellow eel ovaries, which presented few macroscopical changes after in vivo treatment with c-GTH, were as sensitive to gonadotropin in vitro as silver eel ovaries.

\section{Introduction.}

Les ovaires de l'anguille européenne (Anguilla anguilla L.) restent infanfiles pendant que ce poisson est dans nos cours d'eau et n'atteignent leur complet développement qu'au cours de la migration vers lo mer des Sargasses. Malgré une notable augmentation du rapport gonadosomatique (RGS) lors de la transformation des anguilles

Abréviations. - AC : adénylase cyclase : AMPc : 3' 5' adénosine monophosphate cyclique ; EDTA : acide éthylène diamine tétraacétique ; aci-GTH : gonadolropine d'esturgeon ; c-GTH : gonadotropine de carpe ; het-GTH : gonadotropine de poisson-chat indien ; o-LH : lutropine ovine ; o-FSH : follitropine ovine ; RGS : rapport gonadosomatique. 
jaunes sédentaires en anguilles argentées migratrices, les ovocytes de ces dernières ne contiennent pas encore de vitellus. La gonadotropine de carpe (c-GTH) injectée aux anguilles argentées induit un développement des ovaires plus ou moins important selon la durée du traitement (Fontaine et al., 1976).

L'action des gonadotropines, chez les poissons comme chez les mammifères, implique probablement une modification du métabolisme du $3^{\prime} 5^{\prime}$ adénosine monophosphate cyclique (AMPc). En effet, la c-GTH stimule l'adénylate cyclase $(A C)$ dans des homogénats d'ovaire de cyprin (Carassius auratus) (Fontaine et al., 1970) et des extraits d'hypophyse de saumon (Oncorhyncus keta) augmentent la concentration de l'AMPc dans des fragments d'ovaire de truite (Salmo gairdneri), in vitro (Idler ef al., 1975). Dans le cas de l'anguille, le premier type d'expériences $(A C)$ ayant donné des résultats négatifs (Fontaine et al., 1976) nous avons utilisé la seconde approche. Une action de la c-GTH a été effectivement observée, in vitro, sur la concentration de l'AMPc dans l'ovaire d'anguille et divers caractères en onł été précisés.

De plus, nous rapporterons quelques résultats préliminaires concernant l'influence de l'étał physiologique sur l'intensité de la réponse ovarienne à l'hormone, in vitro.

\section{Matériel et méthodes.}

Animaux. - Les anguilles, pesant de 130 d̀ $330 \mathrm{~g}$, proviennent d'étangs de Péronne (Somme) et sont gardées au laboratoire dans des bacs d'eau courante, à l'extérieur.

Hormones. - Nous avons utilisé les gonadotropines de carpe, Cyprinus carpio, (Burzawa-Gérard, 1974), de poisson-chat indien, Heteropneustes fossilis, het-GTH (Burzawa-Gérard et Sundararaj, en préparation) et d'esturgeon, Acipenser stellatus, aci-GTH (Burzawa-Gérard ef al., 1975a) ainsi que la o-LH ef la o-FSH purifiées (Jutisz, CNRS). L'activité de la préparation de LH (M3) est $1,9 \times \mathrm{N} I \mathrm{H}-\mathrm{LH}$ S3 et celle de la préparation de FSH (P 28a) est 8,2 $\times$ NIH-FSH S3.

La poudre acétonique d'hypophyse de carpe a été fournie par les Stoller Fisheries (Spirit Lake, lowa, USA).

Prélèvement ef incubation. - Les anguilles sont sacrifiées par décapitation. Les ovaires sont prélevés en totalité ef pesés pour le calcul du RGS : poids de l'ovaire (g) $\times 100 /$ poids du corps $(\mathrm{g})$. Des lots de 100 à $160 \mathrm{mg}$ de tissu sont préparés, en plusieurs fragments pris dans la partie médiane, sauf dans le cas des anguilles jaunes où il a fallu utiliser la presque totalité de l'ovaire, leur RGS étant plus faible. Ils sont préincubés $\left(20 \mathrm{mn}\right.$, à $20^{\circ} \mathrm{C}$, sous carbogène $\left.95 \mathrm{p} .100 \mathrm{O}_{2}-5 \mathrm{p} .100 \mathrm{CO}_{2}\right)$ dans $4 \mathrm{ml}$ de solution de Krebs-Henseleit bicarbonate $(1 / 2$ en $\mathrm{Ca}) \mathrm{pH} \mathrm{7,4}$ contenant $1 \mathrm{mg} / \mathrm{ml}$ d'albumine, $1 \mathrm{mg} / \mathrm{ml}$ de glucose ef de la théophylline $20 \mathrm{mM}$. L'incubation est faite dans $4 \mathrm{ml}$ de tampon frais contenant éventuellement l'hormone $\left(20 \mathrm{mn}\right.$, à $20^{\circ} \mathrm{C}$, sous carbogène). Chaque essai est réalisé en double.

Extraction ef dosage de l'AMPc. - A la fin de l'incubation, les fragments d'ovaire sont plongés dans $0,5 \mathrm{ml}$ de tampon Tris-HCl $50 \mathrm{mM}$-EDTA $4 \mathrm{mM}$, pH 7,5, à $100^{\circ} \mathrm{C}$ pendant $3 \mathrm{mn}$. L'AMPc de chaque échantillon est extrait de la manière suivante : homogénéisation au Potter à moteur dans les $0,5 \mathrm{ml}$ de Tris-EDTA à $0^{\circ} \mathrm{C}$, suivie de 
deux rinçages de l'appareil $(2 \times 0,25 \mathrm{ml})$; centrifugation à $3500 \mathrm{~g}$ environ, $0^{\circ} \mathrm{C}$, $10 \mathrm{mn}$; prélèvement de la couche intermédiaire entre le culot et les graisses qui surnagent ; puis rinçage du culot par $0,4 \mathrm{ml}$ de Tris-EDTA, centrifugatıon et prélèvement de la couche intermédiaire qui est ajoutée à la première. Le volume total d'extrait( $V$ ) est mesuré et, après une nouvelle centrifugation, on prélève une fraction limpide dont des aliquots de $50 \mu \mathrm{l}$ sont utilisés pour le dosage de l'AMPc. L'AMPc total présent dans l'échantillon est calculé connaissant $V$. L'AMPc est dosé par compétition entre le nucléotide stable inconnu et une quantité donnée d'AMPc tritié pour une protéine spécifique (Gilman, 1970 ; Tovey ef al., 1974). Les réactifs sont fournis par Amersham. Chaque extrait est dosé en double ; la moyenne des deux déterminations constitue le paramètre utilisé.

Variabilité et analyse statistique. - Le coefficient moyen de variation entre les deux déterminations d'AMPc sur le même échantillon de tissu est de 7 p. 100. Le coefficient moyen de variation entre les concentrations d'AMPc dans deux échantillons équivalents d'ovaire est de 10 p. 100.

Les activités de diverses préparations hormonales ont été comparées par analyse de covariance.

\section{Résultats.}

La gonadotropine de carpe augmente considérablement la concentration de l'AMPc dans des fragments d'ovaire. L'intensité de cet effet est indépendante du poids ef du RGS de l'animal (łabl. 1).

\section{TABLEAU 1}

Mise en évidence de l'effet de la c-GTH sur la concentration de l'AMPC dans l'ovaire d'anguille argentée

\begin{tabular}{|c|c|c|c|}
\hline \multirow{2}{*}{$\begin{array}{l}\text { Poids du } \\
\text { corps }(g)\end{array}$} & \multirow{2}{*}{ RGS } & \multicolumn{2}{|c|}{$\begin{array}{c}\mathrm{AMPc}^{*} \\
\text { (pmoles/mg d'ovaire) }\end{array}$} \\
\hline & & sans c-GTH & $\mathrm{c}-\mathrm{GTH}(10 \mu \mathrm{g} / \mathrm{ml})$ \\
\hline $\begin{array}{l}127 \\
160 \\
216 \\
228 \\
290 \\
320 \\
334\end{array}$ & $\begin{array}{l}1,65 \\
1,40 \\
0,98 \\
1,84 \\
1,67 \\
2,16 \\
1,75\end{array}$ & $\begin{array}{l}0,12 \\
0,16 \\
0,14 \\
0,11 \\
0,09 \\
0,20 \\
0,17\end{array}$ & $\begin{array}{l}2,59 \\
2,08 \\
2,84 \\
1,76 \\
2,77 \\
2,18 \\
4,05\end{array}$ \\
\hline
\end{tabular}

* Chaque valeur est la moyenne de déterminations sur deux échantillons de tissus.

Pendant l'incubation (entre 0 et $20 \mathrm{mn}$ ), la concentration de l'AMPc des fragments de tissu ne varie pas notablement en absence d'hormone. Par contre, en présence de 
c-GTH, le paramètre mesuré croît rapidement pendant $10 \mathrm{mn}$, puis l'accumulation du nucléotide cyclique esł beaucoup plus lente (fig. 1).

Le taux d'AMPc est déjà doublé par $0,01 \mu \mathrm{g} / \mathrm{ml}$ de c-GTH et semble augmenter proportionnellement au logarithme de la concentration hormonale dans l'intervalle considéré $(0,01-10 \mu \mathrm{g} / \mathrm{ml})$ (fig. 2).

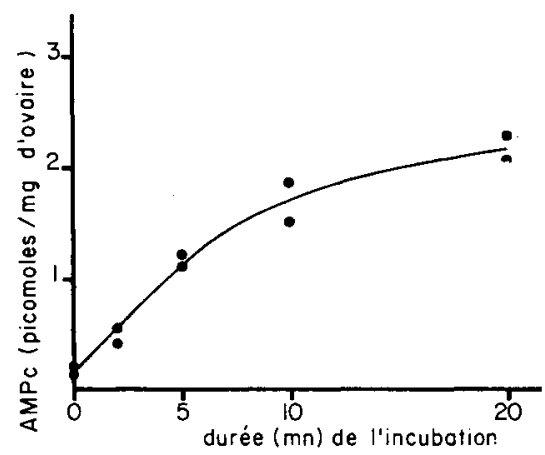

FIG. 1.

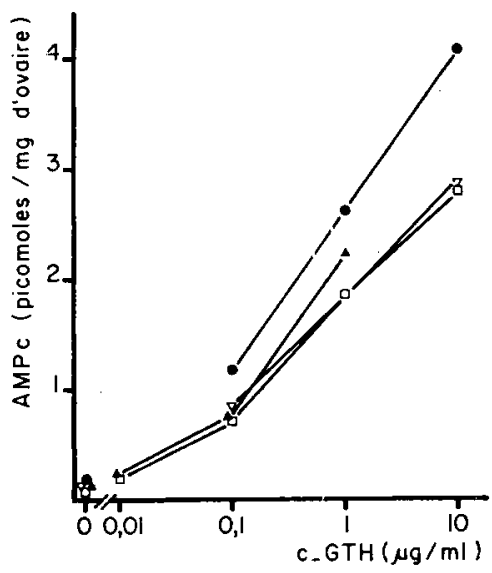

FIG. 2 .

FIG. 1. - Cinétique de l'augmentation de la concentration de l'AMPC dans l'ovaire d'anguille argentée sous l'influence de la c-GTH, in vitro. L'anguille pesait $320 \mathrm{~g}$ et son RGS était égal à 2,16 . La concentration de l'AMPc était $0,16 \mathrm{pmole} / \mathrm{mg}$ d'ovaire au temps 0 ef $0,20 \mathrm{pmole} / \mathrm{mg}$ d'ovaire après une incubation de $20 \mathrm{mn}$ sans hormone. La concentration hormonale utilisée était $10 \mu \mathrm{g} / \mathrm{ml}$.

FIG. 2. - Effet de la concentration de la c-GTH sur la concentration de l'AMPc dans l'ovaire d'anguille orgentée. Les animaux pesaient $=\mathrm{q}: 290 \mathrm{~g}: \bullet: 334 \mathrm{~g} ; \nabla: 216 \mathrm{~g} ; \wedge: 222 \mathrm{~g}$. Les RGS étaient égaux à : $\square: 167 ; \bullet: 1,75 ; \nabla: 0,98 ; \triangle: 1,81$. Chaque point représente la moyenne des valeurs obtenues avec deux échantillions de tissu.

Deux autres gonadotropines de poissons se sont révélées actives dans notre système : celles du poisson-chat indien, het-GTH, et de l'esturgeon, aci-GTH. L'activité de la het-GTH est très voisine de celle de la c-GTH et les pentes correspondant aux deux régressions ne sont pas significativement différentes $(p<0,05)$. Le rapport d'activité, het-GTH/c-GTH est égal à 0,73 (limites pour $p=0,05: 0,35$ et 1,52) et n'est pas significativement différent de la valeur mesurée par le test de spermiation de la grenouille, Rana esculenta $(0,31$ : limites : 0,18-0,56) (Burzawa-Gérard ef Sundararaj, en préparation). Dans le cas de l'aci-GTH, la droite de régression n'est pas parallèle d̀ celle obtenue avec la c-GTH $(0,822$ vs 0,997$)$. Cette différence, significative étant donné la petitesse de la variance dans chaque groupe, est cependant faible. Nous avons done calculé un rapport d'activité moyen qui est égal à 0,091 (limites : $0,080-0,105$ ) tandis qu'il atteint 2,6 (limites : 1,7-3,6) sur la spermiation de la grenouille.

Enfin, les gonadotropines mammaliennes, o-FSH ef o- $\mathrm{LH}, \mathrm{n}$ 'exercent qu'une action très faible pour des concentrations élevées ; $50 \mu \mathrm{g}$ de o-LH ou de o-FSH par $\mathrm{ml}$, bien qu'augmentant nettement (respectivement de 80 p. 100 et de 45 p. 100) la concentration d'AMPc sont considérablement moins efficaces que $0,1 \mu \mathrm{g}$ de $\mathrm{c}-\mathrm{GTH}$. 
Les gonadotropines mammaliennes sont au moins 500 fois moins actives que celle de carpe.

Le RGS de l'anguille argentée s'accroît au cours d'un traitement gonadotrope in vivo (cf légende du tableau 2) mais la réponse de l'AMPc à la c-GTH, in vitro, est bien moins importante que chez les témoins (tabl. 2).

Tous les résultats rapportés jusqu'ici ont été obtenus sur des anguilles argentées. Le traitement in vivo d'anguilles jaunes entraîne au bout de 50 jours une augmentation significative mais faible du RGS (témoins : 0,42 $\pm 0,06$; traitées : 0,67 $\pm 0,04 ; \mathrm{dl}$ : $10 ; t: 2,34 ; p<0,05)$; cependant, in vitro, la c-GTH augmente la concentration ovarienne de l'AMPc de façon aussi intense chez l'anguille jaune témoin que chez l'anguille argentée (tabl. 2).

\section{TABLEAU 2}

Influence de l'état physiologique de l'ovaire sur l'augmentotion par la c-GTH, in vitro, de la concentration ovarienne de l'AMPC

\begin{tabular}{|c|c|c|c|c|c|}
\hline & \multirow{3}{*}{$\begin{array}{l}\text { Poids du } \\
\text { corps }(g)\end{array}$} & \multirow{3}{*}{ RGS } & \multicolumn{3}{|c|}{ AMPc } \\
\hline & & & \multirow{2}{*}{ sans c-GTH } & \multicolumn{2}{|c|}{$\begin{array}{l}\text { Accroissement (x) } \\
\text { par c-GTH }(\mu \mathrm{g} / \mathrm{ml})\end{array}$} \\
\hline & & & & 0,1 & 10 \\
\hline $\begin{array}{l}\text { Anguilles } \\
\text { argentées } \\
\text { témoins }\end{array}$ & $\begin{array}{c}233 * * * \\
\pm 23 \\
(n=9)\end{array}$ & $\begin{array}{c}1,68 * * * \\
\pm 0,11 \\
(\mathrm{n}=9)\end{array}$ & $\begin{array}{c}0,14 * \\
\pm 0,01 \\
(n=9)\end{array}$ & $\begin{array}{c}6,40 * \\
\pm 0,46 \\
(n=4)\end{array}$ & $\begin{array}{c}19,3 * \\
\pm 2,4 \\
(\mathrm{n}=7)\end{array}$ \\
\hline $\begin{array}{l}\text { Anguilles argentées traitées } \\
\text { à l'hypophyse de carpe in vivo }\end{array}$ & $\begin{array}{l}181 \\
245\end{array}$ & $\begin{array}{l}4,01 \\
17,8\end{array}$ & $\begin{array}{l}0,29 * * \\
0,16 * *\end{array}$ & $\begin{array}{l}1,4^{* *} \\
1,5^{* *}\end{array}$ & $\begin{array}{l}5,7 * * \\
6,9 * *\end{array}$ \\
\hline $\begin{array}{l}\text { Anguilles } \\
\text { jaunes } \\
\text { témoins }\end{array}$ & $\begin{array}{c}152 * * * \\
\pm 11 \\
(n=4)\end{array}$ & $\begin{array}{c}0,48 * * * \\
\pm 0,11 \\
(n=4)\end{array}$ & $\begin{array}{c}0,17^{*} \\
\pm 0,02 \\
(\mathrm{n}=4)\end{array}$ & $\begin{array}{c}7,26 * \\
\pm 1,45 \\
(n=3)\end{array}$ & $\begin{array}{c}24,2 * \\
\pm 2,4 \\
(n=4)\end{array}$ \\
\hline
\end{tabular}

Les anguilles traitées reçoivent, par injection, $2,5 \mathrm{mg}$ d'extrait hypophysaire de carpe trois fois par semaine. Le traitement a duré sept semaines pour l'anguille dont le RGS est 4,01 et seize semaines pour celle dont le RGS a atteint 17,8. Pendant ces périodes, les animaux étaient maintenus à $20^{\circ} \mathrm{C}$.

* Moyenne \pm erreur standard des valeurs obtenues sur plusieurs anguilles ( $n=$ nombre d'anguilles). Chaque valeur est la moyenne d'une double détermination sur deux échantillons de tissu (sauf pour les anguilles jaunes).

** Moyenne des valeurs obtenues sur deux échantillons de tissu.

*** Moyenne \pm erreur standard ( $n=$ nombre d'anguilles).

\section{Discussion.}

Il existe une contradiction apparente entre l'inactivité de la c-GTH sur l'AC dans des homogénats d'ovaire d'anguille (Fontaine et al., 1976) et sa capacité d'augmenter considérablement la concentration en AMPc dans des fragments du même tissu. Diverses hypothèses pouvant en rendre comple ont été précédemment énoncées (Fontaine et al., 1976) et ne seront pas discutées ici. En tout cas, la rapidité de l'effet 
de la c-GTH sur la concentration en AMPc suggère qu'il s'agit bien d'une action primaire de l'hormone.

Le paramètre mesuré ici est très sensible puisqu'il augmente déjà significativement avec $0,01 \mu \mathrm{g} / \mathrm{ml}$ de c-GTH ; sa mesure peut constituer la base d'un dosage biologique de la gonadotropine étant donné qu'il semble augmenter, dans un large intervalle, proportionnellement au logarithme de la concentration hormonale; Idler ef al. (1975) ont utilisé dans ce but un système similaire. L'accroissement de la concentration en AMPc dans l'ovaire sous l'influence d'une stimulation gonadotrope est particulièrement important chez l'anguille $(20 \times)$, plus élevé que ceux observés dans d'autres systèmes (environ $10 \times$ ) (cf. Idler ef al., 1975).

Le rapport d'activité aci-GTH/c-GTH est très différent chez l'anguille et chez la grenouille comme ceci a déjà été noté (Burzawa-Gérard et al., 1975b ; Goncharov ef al., 1975). Il s'agit donc de deux hormones nettement différentes, ce qui est probablement en rapport avec l'éloignement phylogénétique des deux poissons étudiés, un téléostéen ef un chondrostéen. En effet, une telle variation n'est pas observée lorsqu'on compare les hormones de deux téléostéens, la carpe ef le poisson-chat indien.

L'inactivité au moins relative des hormones de mammifères ( $\mathrm{LH}$ et FSH) sur la concentration en AMPC de l'ovaire d'anguille est en accord avec de nombreuses données de la littérature (cf. Fontaine, 1975) obtenues également sur des poissons à ovaire peu développé, en particulier celles concernant la stimulation de l'AC de l'ovaire de cyprin (Fontaine et al., 1970).

La diminution importante de l'action in vitro de la c-GTH provoquée par un traitement préalable in vivo peut être due au développement ovarien per se. Cependant les phénomènes de ce type décrits dans des ovaires de mammifères (Bockaert ef al., 1976 ; Hunzicker-Dunn et Birnbaumer, 1976 ; Conti ef al., 1976) ainsi que des résultats préliminaires obtenus au laboratoire suggèrent que nous observons plutôt l'induction d'un état réfractaire par la dernière injection de c-GTH qui a eu lieu 18 heures avant le sacrifice. Cet état peut correspondre à une saturation des récepteurs, ou, plus vraisemblablement, à une désensibilisation par la c-GTH du système récepteuradénylcyclase.

Puisque, enfin, la c-GTH est aussi active in vitro sur la concentration de l'AMPc chez les anguilles jaunes que chez les anguilles argentées, la raison de la relative inefficacité de l'hormone in vivo chez les premières ne réside pas dans l'absence de récepteurs couplés à l'AC. Cette raison doit être recherchée dans des réactions parallèles (Rasmussen et Goodman, 1977) ou consécutives à la formation de l'AMPc.

Symposium sur la Reproduction des Poissons Poimpont, France, 19-21 septembre 1977.

Remerciements. - Nous sommes vivement reconnaissants à Mme Elisabeth BurzawaGérard qui nous a fourni les hormones gonadotropes purifiées de poisson-chat indien, d'esturgeon et de carpe. Nous remercions également $M$. M. Jutisz pour le don des hormones purifiées o-LH et o-FSH.

Travail subventionné en partie par la DGRST (Biologie de la Reproduction et du Développement ; convention no 77-7-0649). 


\section{Références}

BOCKAERT J., HUNZICKER-DUNN M., BIRNBAUMER L., 1976. Hormone stimulated desenzifisation of hormone-dependent adenyl cyclase. J. biol. Chem., 251, 2653-2663.

BURZAWA-GÉRARD E., 1974. Etude biologique et biochimique de l'hormone gonadotrope d'un poisson téléostéen, la carpe (Cyprinus carpio L.). Mém. Mus. nat. Hist. nat., Sér. A, No 86, Paris.

BURZAWA-GÉRARD E., GONCHAROV B. F., FONTAINE Y. A., 1975a. L'hormone gonadotrope hypophysaire d'un poisson chondrostéen, l'esturgeon (Acipenser stellatus Pall.). I. Purification. Gen. comp. Endocrinol., 27, 289-295.

BURZAWA-GERARD E., GONCHAROV B. F., FONTAINE Y. A., 1975b. Further biochemical studies on carp gonadotropin (c-GTH) ; biochemical and biological comparison of c-GTH and a gonadotropin from Acipenser stellatus Pall. (Chondrostei). Gen. comp. Endocrinol., 29, 498-505.

CONTI M., HARWOOD J. P., HSUEH A. J. W., DUFAU M. L., CATT K. J., 1976. Gonadotropin induced loss of hormone receptors and desensitization of adenylate cyclase in the ovary. J. biol. Chem., 251, 7729-7731.

FONTAINE Y. A., 1975. Hormones in fishes, 139-212. In MALINS D. C., SARGENT J. R., Biochemical and biophysical perspectives in marine biology. Vol. 2, Acad. Press, London.

FONTAINE Y. A., BURZAWA-GÉRARD E., DELERUE-LE BELLE N., 1970. Stimulation hormonale de l'activité adénylcyclasique de l'ovaire d'un poisson téléostéen, le cyprin (Carassius auratus L.). C. R. Acad. Sci. Paris, sér. D, 271, 780-783.

FONTAINE Y. A., LOPEZ E., DELERUE-LE BELLE N., FONTAINE-BERTRAND E., LALLIER F., SALMON C., 1976. Stimulation gonadotrope de l'ovaire chez l'anguille (Anguilla anguilla L.) hypophysectomisée. J. Physiol., Paris, 72, 391-396.

GILMAN A. G., 1970. A protein binding assay for adenosine 3' $5^{\prime}$ cyclic monophosphate. Proc. nat. Acad. Sci. U. S., 67, 305-312.

GONCHAROV B. F., BURZAWA-GÉRARD E., FONTAINE Y. A.. 1975. Etude comparative de l'effet biologique des gonadotropines purifiées de la carpe ef de l'esturgeon. Ontogenesis, 7, 85-89.

HUNZICKER-DUNN M., BIRNBAUMER L., 1976. Adenylyl cyclase activities in ovarian tissues. II. Regulation of responsiveness to LH, FSH and PGE, in the rabbit. Endocrinology, 99, 185-197.

IDLER D. R., HWANG S. J., BAZAR L. S., 1975. Fish gonadotropin(s). I. Bioassay of salmon gonadotropin(s) in vitro with immature trout gonads. Endocr. Res. Commun., 2, 199-213.

RASMUSSEN H., GOODMAN D. B. P., 1977. Relationships between calcium and cyclic nucleotides in cell activation. Physiol. Rev., 57, 421-509.

TOVEY K. C., OLDHAM K. G., WHELAN J. A. M., 1974. A simple direct assay for cyclic AMP in plasma and other biological samples using an improved competitive protein binding technique. Clin. chim. Acta, 56, 221-234. 